\title{
Cloud computing sensitive data protection using multi layered approach
}

\begin{abstract}
Cloud computing is a modern technology and it gives access to the network upon request to required computing resources (network, servers, storage, applications and services). This exciting computing model depends on data traffic and controlled by a third party. Despite the expected savings in infrastructure and the development cost for business flexibility, security is still the biggest challenge for the implementation of computing for many service-based companies. This paper discusses challenges related to data security and privacy implementation in cloud computing environment. A framework is proposed to accommodate the protection of sensitive data in cloud computing environment. This framework consists of three levels authorization, security and privacy and saves and verifies level. The main objective of this paper is to discuss the framework implementation and its architecture verification. It is expected from the research a verified framework to protect private and sensitive data in cloud environment.
\end{abstract}

Keyword: Cloud computing; Security; Privacy; Sensitive data 